ORIGINAL ARTICLE

\title{
Examining the professional career values and career awareness of athlete- students: the sample of the faculty of sports sciences
}

\author{
Enver Döşyılmaz ${ }^{1 \mathrm{ABD}}$, Tayfun Şirin ${ }^{1 \mathrm{BCD}}$ \\ Department of Coaching Education, Faculty of Sport Science, Kahramanmaras Sutcu Imam University, \\ Kahramanmaraş, Turkey
}

\begin{abstract}
Background and Study Aim
\end{abstract}

Material and Methods

Results:

Conclusions:

Keywords:
Athlete-students continue their athletic careers at a professional level during their education, or many try to pursue alternative career paths. While much attention has been given to Athlete-students' academic progress and graduation rates in the literature, little attention has been paid to their attitudes towards career values and career awareness. Therefore, this study aimed to determine the career awareness and career values of athletes-students according to some demographic variables.

This cross-sectional descriptive-correlational study was performed in the Sports Science Faculty of Kahramanmaras Sutcu Imam University of Turkey which forms the universe of the study. A total of 334 (162 female and 172 male) athletes-students who were undergraduate students in the sports sciences faculty of the university participated in the research. The data collection tool in the study "Professional Career Awareness Scale", and "Career Values Scale" was used. T-test and One-Way ANOVA tests were used to perform the differential analysis and Pearson Correlation analysis was used to determine the correlation between variables.

According to the results of the research, it was found that scores of the professional career values and career values of the athletes-students were at a moderate level. It was observed that the students' career values dimensions perception scores differed according to their perception scores and gender, department, class, age variables; there was no difference between the variable of being a licensed athlete and the dimensions. Differences were observed between career awareness dimensions, professional development disposition, and department and age variables. There was no difference between career awareness dimensions and gender, status of being a licensed athlete, and class variable. In addition, it is seen that there is a low and moderate relationship between professional career awareness and career values sub-dimensions.

According to these results, it can be said that due to the lack of standardized measures of athlete-student career development, career awareness and career values are at a moderate level and their perceptions differ according to their demographic characteristics. athlete, student, professional development, profession.

\section{Introduction}

Today, with the changing and developing business life, many important concepts have emerged. Undoubtedly, one of these concepts is the concept of career because young people choose a profession and plan the rest of their lives according to the profession they choose. However, over time, the profession preferred by the individuals in their lives goes beyond being a job and becomes a phenomenon that covers their aims, expectations and skills. Here, the concepts of career and career values become important because the concept of career in its most general definition is expressed as the work experiences, skills, expertise areas and knowledge of individuals gained in time. In addition to this, a large number of career definitions can be made since individuals have different needs, values and talents and personal traits of individuals are at the forefront of the concept of career [1]. When the concept of career value is considered, it is defined as the combinations of individuals' needs, motives, attitudes, values and skills [2]. These combinations develop in line with individuals' personality traits, motives, needs and skills and create professional career values. Since these professional career values are shaped according to individuals' expectations, (c) Enver Döşyılmaz, Tayfun Şirin, 2021 doi:10.15561/20755279.2021.0405 interests and professional experiences, a new concept expressed as career awareness emerges. The concept of career awareness emerges as the most important building block of individuals' career development because after individuals make their career choices, they act with career awareness in determining career opportunities, being aware of talents and being directed and managed correctly in their business life [3]. However, it is seen that there are many sub-titles within the concept of career awareness. These are called basic knowledge, education and skill requirements about the profession, requirements of jobs in their natural environment and expectations about professional choices. Therefore, the concept of career awareness is evaluated as a concept covering individuals' abilities to make plans and researches about their careers, their knowledge about career opportunities and decision making process [4]. In a summary of literature related to occupational careers among former athletes [5]. noted that sport is connected to career success when it increases opportunities to complete academic degrees or develop job-related skills, increases support for growth and development not limited to athletic development and progression, and expands experiences, identities, and abilities unrelated to sports. 
In the last two decades, researchers have conducted many researches to understand academic progress and graduation rates of athlete-students [6] and found that they graduated in consistently higher rates than nonathlete university student [7]. However, this does not mean that they are ready for a career after graduation. In 1987, Kennedy and Dimick stated [8] that more attention should be paid to the career development of athletestudents because these athletes were generally lower in career development criteria when compared with nonathlete students. From this point of view, it can be said that athlete-students may not have time to participate in extracurricular activities such as developing career values and awareness and therefore they generally miss relevant professional development opportunities [9].

Ackerman [10] stated that some of the negative effects of participation of athlete-students in sports as an amateur or professional on career development were lack of time, stress and mental fatigue, low grades as a result of not attending classes. On the other hand, Ackerman [10, pp.45-46] stated being an athlete also had many valuable advantages such as athlete-students' transferrable characteristics, scholarships, strong social relationships and professional network. When the literature is reviewed, it can be seen that there are very few studies conducted by using the concepts of professional career value and career awareness together in university students who are athletes. It can be seen that studies on career value are mostly studies in which career values of participants are examined according to their personality traits or in which university students' career values are examined $[11,12$, 13]. It can be seen that studies conducted on professional career awareness are mostly studies in which career awareness levels of participants studying in different educational levels and different areas have been measured $[14,15,16]$. Therefore, it is very important for athletestudents to start planning career from the first years of their educational lives in the process of getting a job, developing skills about their jobs and adapting easily to their job after their student life ends [17]. This is because career value is formed by the combination of life experiences and professional experiences [18]. For this reason, it is very important to increase the career value and professional career awareness of university students and to lead them in this regard. Therefore, the aim of this study is to examine university students' professional career values and career awareness levels in terms of some variables to make a case analysis and to make some recommendations about the professional career values and career awareness levels of athletes who are university students in line with the results obtained.

\section{Material and Methods}

\section{Participants}

This research is a cross-sectional study and its sample consists of 273 athletes-students, 115 females and 158 males, studying at Kahramanmaraş Sütçü İmam University Faculty of Sports Sciences in Turkey in the spring semester of 2019-2020 academic year.

\section{Research Design}

Data Collection Tools

Survey method was used in this study as data collection tool. "Personal Information Form" was used in the first part of the survey which consisted of three parts, while "Career Awareness Scale (CAS)" was used in the second part and "Career Values Questionnaire (CVQ)" was used in the third part.

Career Awareness Scale (CAS): "Career Awareness Scale" developed by Yaşar and Sunay [3] is a 5-Likert type scale with 4 dimensions and 18 items and it aims to measure the career awareness of university students in the field of sports sciences. Dimensions of career awareness were named as "Professional Development Susceptibility", "Professional Readiness", "Professional Consciousness", "Professional Self-Confidence". The lowest score that can be obtained from the scale is " 18 ", while the highest score is " 90 ". Cronbach Alpha value of the data was found as .921. In the present study, Cronbach Alpha value was found as .855 .

Career Values Questionnaire (CVQ): "Career Values Questionnaire" which was developed by [Schein 19] is a 5-Likert type scale with 5 dimensions and 14 items. These dimensions were formed as follows in terms of career values or career goals. In the first factor, career is a tool for "self-realization", in the second factor, career mediates "creating value"; in the third factor, career is a way to "gain power"; in the fourth factor, career provides "autonomy" to create richness for the individual and in the fifth factor, career is a way to "gain respect" [20]. The scale was adapted into Turkish by Halis [20]. In the present study, Cronbach Alpha value was found as .957 .

\section{Statistical Analysis}

The data of the research were analysed with SPSS 22 statistical package program. Frequency and percentage values were used to determine the demographic characteristics [gender, department, class, age, and Licensed Athlete] of the students in the faculty of sports sciences. In addition to that, arithmetic mean and standard deviation data were presented to determine students' Career Awareness Scale and Career Values levels. In addition to descriptive statistics; Normality analyses of the distribution of the research data were evaluated using the skewness and kurtosis tests. It was decided to use parametric tests since normal distribution was found. T-test was conducted to test whether the difference between the two unrelated sample averages was significant in terms of the variables of gender and Licensed Athlete. At the same time one-way analysis of variance (ANOVA) was used to determine whether there was a significant difference between the variables of department, class and age and whether there was a difference between the averages of the groups. Pearson correlation coefficient was calculated to determine the direction and amount of the correlation between Career Awareness Scale and Career Values levels of students.

\section{Results}

The data collected from the sample, its demographical 
and descriptive information about the of athlete- students were as follows (table 1): $42.1 \%$ female, $57.9 \%$ male; Regarding the class variable: $31.9 \%$ are 1 st grade, $21.2 \%$ (135) are 2nd grade, $24.2 \%$ (65) are 3 rd grade and $22.7 \%$, It was determined that studied in the 4th grade; $44.3 \%$ of the participants study in the coaching department, $44.7 \%$ in the teaching department, $11.0 \%$ in the sports management department. When the age variable distribution was examined, it was determined that $59.0 \%$ of the participants were 18-21 years old, $29.7 \%$ were $22-$ 24 years old, and $11.4 \%$ were 25 and over. $48.4 \%$ of the participants stated that they were licensed athletes and $51.6 \%$ were not licensed athletes.

When Table 2 is examined, the arithmetic means and standard deviations of the sub-dimensions of the Career Assessment Scale are found to be $20.42 \pm 5.51$ in the selfactualization sub-dimension; The benefit creation subdimension is $7.96 \pm 2.17$ '; Power gain sub-dimension; $8.07 \pm 2.06$; Autonomy sub-dimension is $11.39 \pm 3.10$;
The sub-dimension of gaining prestige $7.23 \pm 2.04$ was found to be.

The sub-dimensions and standard deviations of the Professional Career Awareness Scale are: professional development disposition sub-dimension was $21.92 \pm 3.89$; Occupational readiness sub-dimension was $16.07 \pm 2.84$; Occupational consciousness sub-dimension was 16.58 \pm 2.82 ; Occupational self-confidence sub-dimension was found to be $14.92 \pm 2.81$.

When Table 3 is examined, a statistically significant difference was found in the sub-dimensions of selfactualization, creating benefits, autonomy and dignity of the CAS according to the gender variable of the participants $(p<0.05)$. When the arithmetic averages were examined, it was seen that this difference was in favor of male participants. No statistically significant difference was observed in any of the sub-dimensions of CVS $(\mathrm{p}>0.05)$.

When Table 4 is examined, there was no statistically

Table 1. Participants' Demographic Characteristics

\begin{tabular}{|c|c|c|c|c|c|}
\hline \multicolumn{2}{|c|}{ Predictor (Variable) } & Participants' & & $f$ & $\%$ \\
\hline \multirow{2}{*}{\multicolumn{2}{|c|}{ Gender }} & Female & & 115 & 42.1 \\
\hline & & Male & & 158 & 57.9 \\
\hline \multirow{4}{*}{\multicolumn{2}{|c|}{ Class }} & 1st Class & & 87 & 31.9 \\
\hline & & 2nd Class & & 58 & 21.2 \\
\hline & & 3rd Class & & 66 & 24.2 \\
\hline & & 4th Class & & 62 & 22.7 \\
\hline \multirow{3}{*}{\multicolumn{2}{|c|}{ Department }} & Coaching & & 121 & 44.3 \\
\hline & & Physical Education & & 122 & 44.7 \\
\hline & & Sport management & & 30 & 11.0 \\
\hline \multirow{3}{*}{\multicolumn{2}{|c|}{ Age }} & 18-21 Age & & 161 & 59.0 \\
\hline & & 22-24 Age & & 81 & 29.7 \\
\hline & & 25 Age and + & & 31 & 11.4 \\
\hline \multirow{2}{*}{\multicolumn{2}{|c|}{ Licensed Athlete }} & Yes & & 132 & 48.4 \\
\hline & & No & & 141 & 51.6 \\
\hline \multicolumn{2}{|c|}{$\begin{array}{l}\text { Total } \\
\text { Table 2. Descriptive stati }\end{array}$} & & & 273 & 100 \\
\hline Sub Dimensions & $\overline{\mathbf{x}}$ & sd & Min. & & Max. \\
\hline SR & 20.40 & 5.51 & 5.00 & & 25.00 \\
\hline $\mathrm{CB}$ & 7.96 & 2.17 & 2.00 & & 10.00 \\
\hline GP & 8.07 & 2.06 & 2.00 & & 10.00 \\
\hline AMY & 11.39 & 3.10 & 3.00 & & 15.00 \\
\hline ER & 7.23 & 2.04 & 2.00 & & 10.00 \\
\hline CVS TOTAL & 3.93 & 0.57 & 1.00 & & 5.00 \\
\hline PDS & 21.92 & 3.89 & 8.00 & & 30.00 \\
\hline PR & 16.07 & 2.84 & 4.00 & & 20.00 \\
\hline PC & 16.58 & 2.82 & 4.00 & & 20.00 \\
\hline PSC & 14.92 & 2.81 & 4.00 & & 20.00 \\
\hline CAS TOTAL & 3,8618 & ,51578 & 1.61 & & 4.83 \\
\hline
\end{tabular}

*N:273, Self-Realization (SR): Creating Benefit (CB): Gaining Power (GP): Autonomy (ATMY): Earn Reputation (ER): Career Values Scales (CVS): Professional Development Susceptibility (PDS); Professional Readiness PR: Professional Consciousness (PC); Professional Self-Confidence (PSC): Career Awareness Scale (CAS). 
Table 3. CVS and CAS sub-dimension t-test results of the participants in terms of the type of Gender

\begin{tabular}{|c|c|c|c|c|c|c|c|}
\hline Scale & Sub Dimensions & Gender & $\mathbf{N}$ & $\overline{\mathbf{x}}$ & ss & $\mathbf{t}$ & $\mathbf{p}$ \\
\hline \multirow{10}{*}{ ¿ } & \multirow{2}{*}{ SR } & Female & 115 & 19.68 & 5.86 & \multirow{2}{*}{-1.89} & \multirow{2}{*}{0.05} \\
\hline & & Male & 158 & 20.96 & 5.19 & & \\
\hline & \multirow{2}{*}{ CB } & Female & 115 & 7.65 & 2.32 & \multirow{2}{*}{-2.02} & \multirow{2}{*}{0.04} \\
\hline & & Male & 158 & 8.18 & 2.04 & & \\
\hline & \multirow{2}{*}{ GP } & Female & 115 & 7.81 & 2.19 & \multirow{2}{*}{-1.75} & \multirow{2}{*}{0.08} \\
\hline & & Male & 158 & 8.25 & 1.96 & & \\
\hline & \multirow{2}{*}{ AMY } & Female & 115 & 10.76 & 3.38 & \multirow{2}{*}{-2.88} & \multirow{2}{*}{0.00} \\
\hline & & Male & 158 & 11.84 & 2.79 & & \\
\hline & \multirow{2}{*}{ ER } & Female & 115 & 6.91 & 2.30 & \multirow{2}{*}{-2.20} & \multirow{2}{*}{0.02} \\
\hline & & Male & 158 & 7.46 & 1.80 & & \\
\hline \multirow{8}{*}{ 足 } & \multirow{2}{*}{ PDS } & Female & 115 & 21.53 & 3.87 & \multirow{2}{*}{-1.42} & \multirow{2}{*}{0.15} \\
\hline & & Male & 158 & 22.20 & 3.89 & & \\
\hline & \multirow{2}{*}{ PR } & Female & 115 & 15.87 & 2.90 & \multirow{2}{*}{-0.96} & \multirow{2}{*}{0.33} \\
\hline & & Male & 158 & 16.21 & 2.80 & & \\
\hline & \multirow{2}{*}{ PC } & Female & 115 & 16.56 & 2.89 & \multirow{2}{*}{-0.12} & \multirow{2}{*}{0.90} \\
\hline & & Male & 158 & 16.60 & 2.77 & & \\
\hline & \multirow{2}{*}{ PSC } & Female & 115 & 14.82 & 3.10 & \multirow{2}{*}{-0.49} & \multirow{2}{*}{0,62} \\
\hline & & Male & 158 & 15.00 & 2.58 & & \\
\hline
\end{tabular}

*N:273, Self Realization (SR): Creating Benefit (CB): Gaining Power (GP): Autonomy (ATMY): Earn Reputation (ER): Career Values Scales (CVS): Professional Development Susceptibility (PDS); Professional Readiness PR: Professional Consciousness (PC); Professional Self-Confidence (PSC): Career Awareness Scale (CAS).

Table 4. CVS and CAS sub-dimension t-test results of the participants in terms of the type of Licensed Athlete

\begin{tabular}{|c|c|c|c|c|c|c|c|}
\hline Scale & Sub Dimensions & Licensed Athlete & $\mathbf{N}$ & $\overline{\mathbf{X}}$ & $\mathbf{s}$ & $\mathbf{t}$ & p \\
\hline \multirow{10}{*}{$\stackrel{\Omega}{己}$} & \multirow{2}{*}{ SR } & Yes & 132 & 19.80 & 5.87 & \multirow{2}{*}{-1.81} & \multirow{2}{*}{0.07} \\
\hline & & No & 141 & 21.00 & 5.09 & & \\
\hline & \multirow{2}{*}{ CB } & Yes & 132 & 7.75 & 2.21 & \multirow{2}{*}{-1.56} & \multirow{2}{*}{0.11} \\
\hline & & No & 141 & 8.16 & 2.13 & & \\
\hline & \multirow{2}{*}{ GP } & Yes & 132 & 7.85 & 2.04 & \multirow{2}{*}{-1.68} & \multirow{2}{*}{0.09} \\
\hline & & No & 141 & 8.27 & 2.07 & & \\
\hline & \multirow{2}{*}{ AMY } & Yes & 132 & 11.03 & 3.14 & \multirow{2}{*}{-0.97} & \multirow{2}{*}{0.06} \\
\hline & & No & 141 & 11.72 & 3.03 & & \\
\hline & \multirow{2}{*}{ ER } & Yes & 132 & 7.10 & 1.95 & \multirow{2}{*}{-1.83} & \multirow{2}{*}{0.33} \\
\hline & & No & 141 & 7.34 & 2.13 & & \\
\hline \multirow{8}{*}{ 崩 } & \multirow{2}{*}{ PDS } & Yes & 132 & 21.77 & 3.87 & \multirow{2}{*}{-0.61} & \multirow{2}{*}{0,53} \\
\hline & & No & 141 & 22.06 & 3.89 & & \\
\hline & \multirow{2}{*}{ PR } & Yes & 132 & 16.30 & 2.90 & \multirow{2}{*}{1.29} & \multirow{2}{*}{0,19} \\
\hline & & No & 141 & 15.85 & 2.80 & & \\
\hline & \multirow{2}{*}{ PC } & Yes & 132 & 16.66 & 2.89 & \multirow{2}{*}{0.43} & \multirow{2}{*}{0,66} \\
\hline & & No & 141 & 16.51 & 2.77 & & \\
\hline & \multirow{2}{*}{ PSC } & Yes & 132 & 15.01 & 3.10 & \multirow{2}{*}{0.50} & \multirow{2}{*}{0,61} \\
\hline & & No & 141 & 14.84 & 2.58 & & \\
\hline
\end{tabular}

*N:273, Self Realization (SR): Creating Benefit (CB): Gaining Power (GP): Autonomy (ATMY): Earn Reputation (ER): Career Values Scales (CVS): Professional Development Susceptibility (PDS); Professional Readiness PR: Professional Consciousness (PC);Professional Self-Confidence (PSC): Career Awareness Scale (CAS). 
significant difference in any of the sub-dimensions of CVS and CAS according to the variable of being licensed athletes $(\mathrm{p}>0.05)$.

When Table 5 is examined, a statistically significant difference was found in the sub-dimensions of career values, such as self-actualization, creating benefit, gaining strength and autonomy, according to the department variable of the athlete-students $(\mathrm{p}<0.05)$. A statistically significant difference was found in the professional development disposition sub-dimension of the career awareness scale $(p<0.05)$. There was no difference in other dimensions.

When Table 6 is examined, a statistically significant difference was found in CVS' self-actualization, creating benefits, gaining power, gaining autonomy and gaining prestige sub-dimensions according to the class variable of the participants $(\mathrm{p}<0.05)$. No statistically significant difference was found in any of the sub-dimensions of CAS ( $\mathrm{p}>0.05)$.

When Table 7 is examined, a statistically significant difference was found in the CVS' strength gain subdimension according to the age variable of the participants $(\mathrm{p}<0.05)$; no difference was found in the sub-dimensions of self-actualization, creating benefit, gaining autonomy and dignity $(p>0.05)$. A statistically significant difference was found in CAS' professional development disposition sub-dimension according to the age variable $(\mathrm{p}<0.05)$, but no difference was found in the Professional Readiness, Professional Consciousness and Professional SelfConfidence sub-dimensions ( $\mathrm{p}>0.05$ )

When Table 8 is examined, it has been determined that there is generally a low and medium level positive

Table 5. Anova-Test results of participants' CVS and CAS sub-dimensions in terms of the variables of department

\begin{tabular}{|c|c|c|c|c|c|c|c|c|}
\hline Scale & Sub-Dimensions & Department & $\mathbf{N}$ & $\overline{\mathbf{X}}$ & ss & $\mathbf{F}$ & $\mathbf{p}$ & Sig. Diff. \\
\hline \multirow{13}{*}{$\stackrel{\sim}{3}$} & SR & $\begin{array}{l}\text { 1.Coaching } \\
\text { 2.Physical Education } \\
\text { 3.Sport management }\end{array}$ & $\begin{array}{l}122 \\
30\end{array}$ & $\begin{array}{l}21.25 \\
19.21 \\
22.00\end{array}$ & $\begin{array}{l}5.88 \\
3.52\end{array}$ & 5.745 & 0.00 & $\begin{array}{l}1^{*}-2 \\
2^{*}-3\end{array}$ \\
\hline & \multirow{3}{*}{ CB } & 1.Coaching & 121 & 8.14 & 2.27 & \multirow{3}{*}{4.598} & \multirow{3}{*}{0.01} & \multirow{3}{*}{$3 *-2$} \\
\hline & & 2.Physical Education & 122 & 7.58 & 2.16 & & & \\
\hline & & 3.Sport management & 30 & 8.80 & 1.42 & & & \\
\hline & \multirow{3}{*}{ GP } & 1.Coaching & 121 & 8.13 & 2.31 & \multirow{3}{*}{4.639} & \multirow{3}{*}{0.01} & \multirow{3}{*}{$3 *-2$} \\
\hline & & 2.Physical Education & 122 & 7.77 & 1.91 & & & \\
\hline & & 3.Sport management & 30 & 9.03 & 1.12 & & & \\
\hline & \multirow{3}{*}{ AMY } & 1.Coaching & 121 & 11.72 & 3.04 & \multirow{3}{*}{6.327} & \multirow{3}{*}{0.00} & \multirow{3}{*}{$\begin{array}{l}1^{*}-2 \\
3^{*}-2\end{array}$} \\
\hline & & 2.Physical Education & 122 & 10.73 & 3.30 & & & \\
\hline & & 3.Sport management & 30 & 12.70 & 1.46 & & & \\
\hline & \multirow{3}{*}{ ER } & 1.Coaching & 121 & 7.25 & 2.23 & \multirow{3}{*}{2.158} & \multirow{3}{*}{0.11} & \multirow{3}{*}{-} \\
\hline & & 2.Physical Education & 122 & 7.04 & 2.00 & & & \\
\hline & & 3.Sport management & 30 & 7.90 & 1.09 & & & \\
\hline \multirow{12}{*}{ 尔 } & \multirow{3}{*}{ PDS } & 1.Coaching & 121 & 21.48 & 3.97 & \multirow{3}{*}{13.575} & \multirow{3}{*}{0.00} & \multirow{3}{*}{$\begin{array}{l}3^{*}-2 \\
3^{*}-1\end{array}$} \\
\hline & & 2.Physical Education & 122 & 21.53 & 3.80 & & & \\
\hline & & 3.Sport management & 30 & 25.26 & 1.87 & & & \\
\hline & \multirow{3}{*}{$P R$} & 1.Coaching & 121 & 15.84 & 3.04 & \multirow{3}{*}{1.039} & \multirow{3}{*}{0.35} & \multirow{3}{*}{-} \\
\hline & & 2.Physical Education & 122 & 16.16 & 2.77 & & & \\
\hline & & 3.Sport management & 30 & 16.63 & 2.22 & & & \\
\hline & \multirow{3}{*}{ PC } & 1.Coaching & 121 & 16.66 & 2.94 & \multirow{3}{*}{0.236} & & \\
\hline & & 2.Physical Education & 122 & 16.46 & 2.80 & & 0.79 & - \\
\hline & & 3.Sport management & 30 & 16.80 & 2.36 & & & \\
\hline & & 1.Coaching & 121 & 14.77 & 2.68 & & & \\
\hline & PSC & 2.Physical Education & 122 & 15.17 & 2.95 & 0.929 & 0.39 & - \\
\hline & & 3.Sport management & 30 & 14.53 & 2.75 & & & \\
\hline
\end{tabular}

The groups in favor of the significant difference are shown with $\left({ }^{*}\right) .{ }^{*} \mathrm{~N}: 273$, Self Realization (SR):Creating Benefit (CB): Gaining Power (GP): Autonomy (ATMY): Earn Reputation (ER): Career Values Scales (CVS): Professional Development Susceptibility (PDS); Professional Readiness PR: Professional Consciousness (PC); Professional Self-Confidence (PSC): Career Awareness Scale (CAS); 
relationship between the sub-dimensions of CVS and CAS.

\section{Discussion}

As a result of the analysis of data obtained from the study, male participants' perceptions of self-realization, creating value, autonomy and gaining respect sub- dimensions of career values were found to be high. No difference was found in terms of gender in any subdimensions of professional career awareness scale (Table 3). In their study conducted to find out the factors affecting career values, Başol, et al., [21]; Koca [22]; Pala [23] found differences between the variable of gender and career values sub-dimension in favor of male participants.

Table 6. Anova-Test results of participants' CVS and CAS sub-dimensions in terms of the variables of Class

\begin{tabular}{|c|c|c|c|c|c|c|c|c|}
\hline Scale & Sub Dimensions & Class & $\mathbf{N}$ & $\overline{\mathbf{x}}$ & ss & $\mathbf{F}$ & $\mathbf{p}$ & Sig. Diff. \\
\hline \multirow{20}{*}{$\stackrel{\sim}{\mho}$} & \multirow{4}{*}{ SR } & 1st Class & 87 & 22.26 & 4.85 & \multirow{4}{*}{8.303} & \multirow{4}{*}{0.00} & \multirow{4}{*}{$\begin{array}{l}1^{*}-4 \\
3^{*}-4\end{array}$} \\
\hline & & 2nd Class & 58 & 20.00 & 5.86 & & & \\
\hline & & 3rd Class & 66 & 20.72 & 4.89 & & & \\
\hline & & 4th Class & 62 & 17.91 & 5.75 & & & \\
\hline & \multirow{4}{*}{ CB } & 1st Class & 87 & 8.54 & 1.93 & \multirow{4}{*}{6.966} & \multirow{4}{*}{0.00} & \multirow{4}{*}{$\begin{array}{l}1^{*}-4 \\
3^{*}-4\end{array}$} \\
\hline & & 2nd Class & 58 & 7.86 & 2.56 & & & \\
\hline & & 3rd Class & 66 & 8.21 & 1.62 & & & \\
\hline & & 4th Class & 62 & 6.98 & 2.32 & & & \\
\hline & \multirow{4}{*}{ GP } & 1st Class & 87 & 8.64 & 1.97 & \multirow{4}{*}{6.622} & \multirow{4}{*}{0.00} & \multirow{4}{*}{$\begin{array}{l}1 *-4 \\
3 *-4\end{array}$} \\
\hline & & 2nd Class & 58 & 7.93 & 2.51 & & & \\
\hline & & 3rd Class & 66 & 8.27 & 1.49 & & & \\
\hline & & 4th Class & 62 & 7.19 & 2.00 & & & \\
\hline & \multirow{4}{*}{ AMY } & 1st Class & 87 & 12.13 & 2.74 & \multirow{4}{*}{9.533} & \multirow{4}{*}{0.00} & \multirow{4}{*}{$\begin{array}{l}1^{*}-4 \\
3^{*}-4\end{array}$} \\
\hline & & 2nd Class & 58 & 11.06 & 3.40 & & & \\
\hline & & 3rd Class & 66 & 12.18 & 2.49 & & & \\
\hline & & 4th Class & 62 & 9.80 & 3.26 & & & \\
\hline & \multirow{4}{*}{ ER } & 1st Class & 87 & 7.50 & 2.01 & \multirow{4}{*}{8.138} & \multirow{4}{*}{0.00} & \multirow{4}{*}{$\begin{array}{l}1 *-4 \\
3 *-2 \\
3 *-4\end{array}$} \\
\hline & & 2nd Class & 58 & 6.84 & 2.44 & & & \\
\hline & & 3rd Class & 66 & 7.98 & 1.27 & & & \\
\hline & & 4th Class & 62 & 6.40 & 2.02 & & & \\
\hline \multirow{16}{*}{ ভ্ড } & \multirow{4}{*}{ PDS } & 1st Class & 87 & 21.50 & 4.45 & \multirow{4}{*}{2.407} & \multirow{4}{*}{0.06} & \multirow{4}{*}{-} \\
\hline & & 2nd Class & 58 & 21.15 & 3.62 & & & \\
\hline & & 3rd Class & 66 & 22.78 & 3.38 & & & \\
\hline & & 4th Class & 62 & 22.30 & 3.66 & & & \\
\hline & & 1st Class & 87 & 15.54 & 3.34 & & & \\
\hline & PR & 2nd Class & 58 & 15.93 & 2.77 & 2132 & רח & - \\
\hline & 政 & 3rd Class & 66 & 16.62 & 2.24 & 2.102 & J & 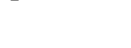 \\
\hline & & 4th Class & 62 & 16.37 & 2.65 & & & \\
\hline & & 1st Class & 87 & 16.37 & 3.53 & & & \\
\hline & $P C$ & 2nd Class & 58 & 16.31 & 2.80 & 1089 & 035 & _ \\
\hline & 10 & 3rd Class & 66 & 17.10 & 2.04 & & & \\
\hline & & 4th Class & 62 & 16.59 & 2.37 & & & \\
\hline & & 1st Class & 87 & 14.94 & 3.07 & & & \\
\hline & PSC & 2nd Class & 58 & 14.17 & 2.82 & 2169 & 009 & _- \\
\hline & & 3rd Class & 66 & 15.42 & 2.70 & & & \\
\hline & & 4th Class & 62 & 15.08 & 2.43 & & & \\
\hline
\end{tabular}

The groups in favor of the significant difference are shown with $\left({ }^{*}\right) .{ }^{*} \mathrm{~N}: 273$, Self Realization (SR): Creating Benefit (CB): Gaining Power (GP): Autonomy (ATMY): Earn Reputation (ER): Career Values Scales (CVS): Professional Development Susceptibility (PDS); Professional Readiness PR: Professional Consciousness (PC); Professional Self-Confidence (PSC): Career Awareness Scale (CAS). 
Table 7. Anova-Test results of participants' CVS and CAS sub-dimensions in terms of the variables of Age

\begin{tabular}{|c|c|c|c|c|c|c|c|c|}
\hline Scale & Sub Dimensions & Age & $\mathbf{N}$ & $\overline{\mathbf{x}}$ & ss & $\mathbf{F}$ & $p$ & Sig. Diff. \\
\hline \multirow{15}{*}{$\stackrel{\sim}{3}$} & \multirow{3}{*}{ SR } & 1. $18-21$ & 161 & 20.71 & 5.49 & \multirow{3}{*}{2.613} & \multirow{3}{*}{0.07} & \multirow{3}{*}{-} \\
\hline & & 2. $22-24$ & 81 & 19.35 & 5.87 & & & \\
\hline & & 3. 25 and above & 31 & 21.70 & 4.10 & & & \\
\hline & \multirow{3}{*}{$\mathrm{CB}$} & 1. $18-21$ & 161 & 8.01 & 2.19 & \multirow{3}{*}{1.588} & \multirow{3}{*}{0.20} & \multirow{3}{*}{-} \\
\hline & & 2. $22-24$ & 81 & 7.66 & 2.30 & & & \\
\hline & & 3. 25 and above & 31 & 8.45 & 1.60 & & & \\
\hline & \multirow{3}{*}{ GP } & 1. $18-21$ & 161 & 8.19 & 2.10 & \multirow{3}{*}{3.003} & \multirow{3}{*}{0.05} & \multirow{3}{*}{$3^{*}-2$} \\
\hline & & 2. $22-24$ & 81 & 7.64 & 2.15 & & & \\
\hline & & 3. 25 and above & 31 & 8.58 & 1.43 & & & \\
\hline & \multirow{3}{*}{ ATMY } & 1. $18-21$ & 161 & 11.42 & 3.12 & \multirow{3}{*}{1.163} & \multirow{3}{*}{0.31} & \multirow{3}{*}{-} \\
\hline & & 2. $22-24$ & 81 & 11.00 & 3.34 & & & \\
\hline & & 3. 25 and above & 31 & 12.06 & 2.15 & & & \\
\hline & \multirow{3}{*}{ ER } & 1. $18-21$ & 161 & 7.31 & 2.05 & \multirow{3}{*}{1.741} & \multirow{3}{*}{0.17} & \multirow{3}{*}{-} \\
\hline & & 2. $22-24$ & 81 & 6.91 & 2.20 & & & \\
\hline & & 3. 25 and above & 31 & 7.64 & 1.45 & & & \\
\hline \multirow{12}{*}{ 芩 } & \multirow{3}{*}{ PDS } & 1. $18-21$ & 161 & 21.49 & 3.81 & \multirow{3}{*}{5.051} & \multirow{3}{*}{0.00} & \multirow{3}{*}{$\begin{array}{l}3 *-1 \\
3 *-2\end{array}$} \\
\hline & & 2. $22-24$ & 81 & 22.03 & 3.91 & & & \\
\hline & & 3. 25 and above & 31 & 23.87 & 3.73 & & & \\
\hline & \multirow{3}{*}{ PR } & 1. $18-21$ & 161 & 15.93 & 2.91 & \multirow{3}{*}{0.886} & \multirow{3}{*}{0.41} & \multirow{3}{*}{-} \\
\hline & & 2. $22-24$ & 81 & 16.11 & 2.86 & & & \\
\hline & & 3. 25 and above & 31 & 16.67 & 2.42 & & & \\
\hline & \multirow{3}{*}{$P C$} & 1. $18-21$ & 161 & 16.62 & 2.81 & & & \\
\hline & & 2. $22-24$ & 81 & 16.28 & 2.99 & 1.202 & 0.30 & - \\
\hline & & 3. 25 and above & 31 & 17.19 & 2.27 & & & \\
\hline & & 1. $18-21$ & 161 & 15.03 & 2.66 & & & \\
\hline & PSC & 2. $22-24$ & 81 & 14.76 & 3.13 & 0.301 & 0.74 & - \\
\hline & & 3. 25 and above & 31 & 14.77 & 2.75 & & & \\
\hline
\end{tabular}

The groups in favor of the significant difference are shown with $\left({ }^{*}\right) .{ }^{*} \mathrm{~N}: 273$, Self Realization (SR): Creating Benefit (CB): Gaining Power (GP): Autonomy (ATMY): Earn Reputation (ER): Career Values Scales (CVS): Professional Development Susceptibility (PDS); Professional Readiness PR:Professional Consciousness (PC); Professional Self-Confidence (PSC): Career Awareness Scale (CAS).

These results support the study results. Unlike the results of the present study, in their studies they examined the factors affecting career values of associate degree students Şentürk and Buran [24] reported that female participants had higher perceptions between the variable of gender and career values. Eratlı Şirin and Aydın [25] examined the career values of students in the faculty of sports sciences and reported that there were no differences between the variable of gender and sub-dimensions of career values scale. Dinç [14, pp:139] and Öztürk [15, pp. 78-79] examined the career awareness of university students and did not find any differences between the variable of gender and career awareness Lacole [26] found that female athlete-students had higher career awareness and they may have had more diverse and broader past experiences guiding them in making career decisions.
Another result of the study was that no differences were found between the variable of being a licensed athlete and career values and sub-dimensions of professional career awareness (Table 4). There are studies in literature which show that being a licensed athlete has no effect on career development [27]. When the literature on career values is examined, in their study examining career values of students of sports sciences faculty, Eratl Şirin and Aydın [25, p.42] found perceptions of female athletes higher. This result was found to be different than the results of our study. In a study on career differences, Dinç [14, p.140] reported that there were no differences between the variable of being an active athlete and career awareness.

In terms of the departments of students, it was found that in general students in the department 
Table 8. Pearson's Correlation Analysis Results of CVS and CAS sub-dimensions

\begin{tabular}{rlllll}
\hline \multicolumn{1}{c}{ Scale } & CAS & & & \\
\hline & Sub Dimensions & PDS & PR & PC & PSC \\
& SR & $0.27^{* *}$ & $0.31^{* *}$ & $0.46^{* *}$ & $0.24^{* *}$ \\
& CB & $0.23^{* *}$ & $0.28^{* *}$ & $0.38^{* *}$ & $0.19^{* *}$ \\
& GP & $0.22^{* *}$ & $0.31^{* *}$ & $0.45^{* *}$ & $0.21^{* *}$ \\
& AMY & $0.25^{* *}$ & $0.33^{* *}$ & $0.36^{* *}$ & $0.33^{* *}$ \\
& ER & $0.25^{* *}$ & $0.28^{* *}$ & $0.40^{* *}$ & $0.26^{* *}$ \\
\hline
\end{tabular}

$\mathrm{n}=273^{* *}$. Correlation is significant at the 0.01 level [2-tailed]. Self Realization (SR):Creating Benefit (CB): Gaining Power (GP): Autonomy (ATMY): Earn Reputation (ER): Career Values Scales (CVS): Professional Development Susceptibility (PDS); Professional Readiness PR: Professional Consciousness (PC); Professional Self-Confidence (PSC): Career Awareness Scale (CAS).

of sport management had higher career values and professional development tendency than the students of other departments. The reason for this result may be the fact that in general students in the department of sport management prefer this department as the second university and therefore they have higher career values and career awareness. Eratlı Şirin et.al [28], in their study on the social entrepreneurship of the students of the faculty of sports sciences, concluded that the dimensions of risk taking and self-confidence that affect the career awareness of the students are high. When the literature on career values is examined, Koca [22, pp.59]; Şentürk and Buran [24, pp.168]; [Eratlı Şirin and Aydın [25, pp.325] reported difference between career values and academic departments in career choice. In their study they examined the career values of university student athletes, Karakaya et al., [29] reported that there were no differences between the participants' faculties and career values. When the literature on professional career awareness was examined, in a study which examined the effect of university career development program on students' career awareness, Öztürk [15, pp.85] reported difference between participants' faculties and career awareness levels; while Dinç [14, pp.145] did not report any differences between the variable of department and career values. It can be seen that these results are different from study results. As a result of the study, it was found that student athletes in their first year of study had higher career values than the other students, while no difference was found in career awareness. It can be said that they have high career values since they are in the first year of their education life studying in a department they chose and it can be said that since students choose their careers, they are aware of their talents and they will act with career awareness from then on. Eratlı Sirin and Aydin [25, pp.227] reached similar results. When the literature about professional career awareness was examined, Öztürk [15, pp.90] found that participants' career awareness levels increased as their year of study increased. As another result of the study, it was found that older students had higher career values gaining power sub-dimension perceptions and professional development tendency sub-dimension in professional career awareness.
In their studies examining the factors affecting career values of students, Şentürk and Buran [24, pp.170]; Eratlı Şirin and Aydın [25, pp.327] examined the differences between the variable of age and career values and found that participants who were 25 and older had higher career value perceptions than the participants who were 20 and younger. When the literature about professional career awareness was examined, Öztürk [15, pp.90] found that university students who were 26 and older had higher career awareness levels than students who were younger.

\section{Conclusion}

The results of the study showed a low and moderate positive association between career values scale and professional career awareness scale sub-dimensions (Table 8 ). As a result of this analysis, a direct proportion was found between the participants' career value perceptions and professional career awareness levels.

As a conclusion, it was found that while the variables of gender, department, year of study and age were partly effective on career values and professional career awareness, the variables of being licensed and monthly income did not have any effects on career values and professional career awareness. In addition, it was found that university student athletes' professional career awareness levels increased as their career values perceptions increased.

Based on the results of the research, it can be suggested that the instructors guide and support the students in order to increase the career values and awareness of the students. In their research, Eratlı Şirin and Şahin [30] stated that the creation of a school environment based on the counseling of instructors in institutions that train physical education teachers is a very important factor in terms of mutual trust, communication and support, which students need, in terms of awareness of students.

\section{Conflict of interests}

The authors state that there is no conflict of interest 


\section{References}

1. Schein, EH. Career Anchors and Career Paths: A Panel Study of Management School Graduates. Cambridge: Organizational Studies Group, Sloan School of Management, Massachusetts Institute of Technology; 1974.

2. Batur HZ, Adıgüzel O. A research based on factors effectıng students' carrier decisions from the perspective of schein's carrier anchors: sample case of science high schools in the city of Isparta. Dumlupinar University Journal of Social Sciences, 2014; (42): 327-348.

3. Yaşar O, Sunay H. Development of sports awareness scale: validity and reliability study. The Journal of Physical Education and Sport Sciences, 2020; 18(1): 46-58. https://doi.org/10.33689/spormetre.672441

4. Nasir R, Lin LS. The relationship between selfconcept and career awareness amongst students. Asian Social Science, 2013; 9(1): 193-197. https://doi.org/10.5539/ass.v9n1p193

5. Coakley J. Sports in society: Issues and controversies. New York, NY: McGraw-Hill Education; 2015.

6. Paskus TS. A summary and commentary on the quantitative results of current NCAA academic reforms. Journal of Intercollegiate Sport, 2012; 5: 41-53. https://doi.org/10.1123/jis.5.1.41

7. Petr TA, McArdle JJ. Academic research and reform: A history of the empirical basis for NCAA academic policy. Journal of Intercollegiate Sport, 2012; 5: 27-40. https://doi.org/10.1123/jis.5.1.27

8. Kennedy SR, Dimick KM. Career maturity and professional sports expectations of college football and basketball players. Journal of College Student Personnel, 1987; 28(4): 293-297.

9. Weisberg, S. NCAA survey delves into practice time, coaches' trust. USA Today. [Internet]. 2011 [updated 2010 Jan 10; cited 2020 Apr 10]. Available from: http://usatoday30.usatoday. com/sports/college/2011-01-14-ncaa-survey N.htm

10.Ackerman C. Exploration of Factors Related to Development of Vocational Identity in Collegiate Student Athletes: (615202013-001) 2013. https://doi.org/10.1037/e615202013-001

11. Bayram N, Gürsakal S, Aytaç S. The Influence of Personality on Students' Career Values. Journal of Social Sciences, 2012; 12(2): 181-189.

12.Kim J, Lee M. The Effects of career development based on university \& college students' work experience on career values. The Journal of Career Education Research, 2018; 31(3): 135-157. https://doi.org/10.32341/jcer.2018.09.31.3.135

13.Jackson D, Tomlinson M. Career values and proactive career behaviour among contemporary higher education students. Journal of Education and Work, 2019;32(2):449-464. https://doi.org/10.1080/13639080.2019.1679730

14. Dinç A. Investigating the relationship between ethical values and career awareness of Iğdır University sports science students. African Educational Research Journal (Special Issue), 2020; 8(2): 137-141. https://doi.org/10.30918/AERJ.8S2.20.040

15.Öztürk M. The effects of university career development program on students' career awareness, decision-making self-efficacy, and stres. Istanbul: Marmara Üniversity. Press; 2020.
16.Lee J, Kim A. An exploratory study on the career awareness of high school students: Focusing on the impact of cultural capital and social capital. The journal of Educational Studies, 2020; 51(3): 171-191. https://doi.org/10.15854/jes.2020.09.51.3.171

17.Akoğlan Kozak M, Dalkıranoğlu T. Career perceptions of new graduates: Anadolu University Example, Anadolu University Journal of Social Sciences Institute, 2013; 13(1): 41-52.

18.Erdoğmuş N. Career development theory and practice. Ankara: Nobel Publishing. Press; 2003.

19.Schein EH. Career Anchors Revisited: Implications for career development in the 21th century, Academy of Management Executive, 1996;10(4): 80-88. https://doi.org/10.5465/ame.1996.3145321

20.Halis M, Cumaliyeva D. The effects of spiritual values on career values in career choice: A research on students, Route Educational and Social Science Journal, 2016; 3(2):17-30. https://doi.org/10.17121/ressjournal.514

21.Başol O, Bilge E, Kuzgun Ş. A research for determining of career values of students: Personal values, Ejovoc (Electronic Journal of Vocational Colleges, 2012; 2(2): 57-68.

22.Koca Aİ. The relationship of students' career preferences to personal values and demographic characteristics at Cukurova University, Çukurova University Faculty of Economics and Administrative Sciences, 2010; 14(1): 56-70. https://doi.org/10.25204/iktisad.429513

23.Pala A. Factors of affecting students' career values: The example of faculty of sports science, International Journal of Human Sciences, 2016; 13(1): 1897-1905. https://doi.org/10.14687/ijhs.v13i1.3707

24.Şentürk EE, Buran KA. Study on factors affecting the associate degree student's career anchors, Ejovoc (Electronic Journal of Vocational Colleges), 2015; 5(1): 162-180.

25.Eratlı Şirin, Y, Aydın Ö. Investigation of career values of faculty of sports sciences students, Mediterranean Journal of Sport Science, 2021; 3(2): 317-330. https://doi.org/10.38021/asbid.833906

26.Lacole LH. Athletic Identity, Vocational Identity, and Occupational Engagement in College Student-Athletes and Non-Athletes. Kansas. Kansas Universty, Press; 2010.

27.Finch J. The importance of clinical academic careers in nursing. Journal of Research in Nursing, 2009; 14(2): 103-105. https://doi.org/10.1177/1744987108101143

28.Eratlı Şirin Y, Bilir FP, Öz G. Universty students social entrepreneurship trends: The example of school of physical education and sport, Gaziantep University Journal of Sports Sciences, 2018; 3(3): 35-47.

29.Karakaya YE, Karataș Ö, Özdenk C, Karataş F. University sports students' perceptions of career value, Dogus University Journal, 2013; 14(1): 86-94. https://doi.org/10.31671/dogus.2018.98

30.Eratl1 Şirin Y, Şahin M. Investigation of factors affecting the achievement of university students with logistic regression analysis: School of physical education and sport example, SAGE Open, 2020:1-9. https://doi.org/10.1177/2158244020902082 


\section{Information about the authors:}

Enver Döşyilmaz; https://orcid.org/0000-0001-6268-096X; dosyilmaz@ksu.edu.tr; Department of Coaching Education, Faculty of Sport Science, Kahramanmaras Sutcu Imam University, Kahramanmaraş, Turkey.

Tayfun Şirin; (Corresponding Author); https://orcid.org/0000-0001-6305-0097; tayfunksu@gmail.com; Department of Coaching Education, Faculty of Sport Science, Kahramanmaras Sutcu Imam University, Kahramanmaraş, Turkey

\section{Cite this article as:}

Döşyılmaz E, Şirin T. Examining the professional career values and career awareness of athlete- students: the sample of the faculty of sports sciences. Physical Education of Students, 2021;25(4):239-248.

https://doi.org/10.15561/20755279.2021.0405

This is an Open Access article distributed under the terms of the Creative Commons Attribution License, which permits unrestricted use, distribution, and reproduction in any medium, provided the original work is properly cited http://creativecommons.org/licenses/by/4.0/deed.en

Received: 10.07.2021

Accepted: 14.08.2021; Published: 30.08.2021 